\title{
Interactions of primary neuroepithelial progenitor and brain endothelial cells: distinct effect on neural progeni- tor maintenance and differentiation by soluble factors and direct contact
}

\author{
Miguel A Gama Sosa ${ }^{1,3}$, Rita De Gasperi ${ }^{1,3}$, Anne B Rocher ${ }^{2}$, Gissel M Perez ${ }^{1,3}$, Keila Simons ${ }^{1,3}$, Daniel E Cruz ${ }^{1,3}$, \\ Patrick R Hof ${ }^{2}$, Gregory A Elder ${ }^{1,3}$ \\ Departments of ${ }^{l}$ Psychiatry and ${ }^{2}$ Neuroscience, Mount Sinai School of Medicine of New York University, New York, NY and ${ }^{3}$ Research \\ and Development Service, James J. Peters Department of Veterans Affairs Medical Center, 130 West Kingsbridge Road, Bronx, New \\ York 10468, USA
}

Neurovascular interactions are crucial for the normal development of the central nervous system. To study such interactions in primary cultures, we developed a procedure to simultaneously isolate neural progenitor and endothelial cell fractions from embryonic mouse brains. Depending on the culture conditions endothelial cells were found to favor maintenance of the neuroprogenitor phenotype through the production of soluble factors, or to promote neuronal differentiation of neural progenitors through direct contact. These apparently opposing effects could reflect differential cellular interactions needed for the proper development of the brain.

Keywords: differentiation, culture, endothelial cells, neuroepithelial cells

Cell Research (2007) 17:619-626. doi: 10.1038/cr.2007.53; publication online 26 June 2007

\section{Introduction}

Endothelial cells (EC) affect the growth and differentiation of many cell types in diverse organs including the nervous system. Vascular development is closely associated with the development of the nervous system through the formation of closed neurovascular networks that require the ordered and coordinated action of signaling factors targeting EC and neurons. During development, differentiation of mesenchymal cells into EC leads to the formation of a primary vascular plexus that is later remodeled into a highly branched hierarchical vascular tree composed of capillaries, arteries and veins. Vascular development of the brain begins with the formation of a capillary network in the head mesenchyme. The brain vascular system then

Correspondence: Miguel A Gama Sosa

Tel: +718-584-9000 ext 1839; Fax: +718-562-9120

E-mail: miguel.gama-sosa@mssm.edu

Received 12 June 2006; revised 4 July 2006; accepted 13 February 2007; published online 26 June 2007 develops through the sprouting of capillaries into the brain parenchyma [1]. In the brain as in other organs this maturation process involves recruitment of mural cells, development of a surrounding matrix and elastic lamina, as well as eventually brain-specific specialization of EC to form the tight junctions found in the adult blood-brain barrier [1].

Recently there has been much interest in the similarities between development of the vascular system and nervous system and particularly in the influences of neuroepithelial cells on vascular EC and vice versa [2-7]. For example in the developing brain, neuroepithelial cells in the ventricular zone produce vascular endothelial growth factor (VEGF), which attracts vessel growth into the brain [8]. In addition, many proangiogenic factors (such as insulin like growth factor-1 (IGF-1), basic fibroblast growth factor (bFGF), interleukin-8 (IL-8), erythropoietin and angiopoietin-1) that promote the recruitment, proliferation, and survival of EC also promote the proliferation of neural progenitors, neurogenesis, synaptogenesis, axonal growth and neuroprotection [5]. Conversely many neurogenic factors 
including nerve growth factor (NGF), brain derived nerve growth factor (BDNF), neuropilin, glial derived growth factor (GDNF) and artermin affect both neurons and EC $[5,7]$. These interactions are not limited to embryogenesis as evidenced by the finding that neural stem cells in the adult hippocampus and subventricular zone lie in close apposition to blood vessels [9]. Recent in vitro studies have suggested that within this vascular niche, EC stimulate the self-renewal of neural stem cells by preventing their differentiation [10].

As vessels grow into the embryonic brain, EC could interact with brain neuroepithelial cells through secreted soluble factors (long range interactions) or through direct physical contact (short range interactions) with potentially different biological effects. Using primary cultures we examined the effect of EC on neural progenitor growth and differentiation using different co-culture conditions. Depending on the co-culture system, EC were found to either promote maintenance of the neural progenitor state via soluble factors or to promote neuronal differentiation by direct contact.

\section{Materials and Methods}

Isolation of neural progenitor and vascular endothelial cells.

Primary EC were isolated from E13.5-14.5 embryonic brain. After removal of the meningeal tissues, the cerebral cortices and the mesencephalon were dissected and separately dissociated at room temperature in $1 \mathrm{ml}$ of $0.006 \%$ trypsin (Invitrogen, Calsbad, CA) in phosphate buffered saline (PBS) using fire-polished Pasteur pipettes. After the addition of one volume of complete DMEM (DMEM supplemented with $20 \%$ heat-inactivated fetal calf serum, penicillin, streptomycin, glutamine and fungizone), the dissociated cells were centrifuged at $1000 \times g$ for $5 \mathrm{~min}$ and resuspended in complete DMEM containing $40 \mathrm{U} / \mathrm{ml}$ deoxyribonuclease I (DNase I, Sigma-Aldrich, St. Louis, MO). The neural fraction was filtered through a $75 \mu \mathrm{m}$ nylon mesh, and kept on ice for later use in the co-culture experiments. The microvessel fraction retained on the mesh was washed three times with PBS and dislodged by inverting the mesh and rinsing with PBS. To partially liberate the EC, the microvessel fraction (cortical and mesencephalic pool to increase the yield) was digested with 0.025 units $/ \mathrm{ml}$ of collagenase (Vibrio alginolyticus) and 0.2 units/ml dispase (Bacillus polymyxa) (Roche, Indianapolis, IN) for 1-2 min at room temperature in $0.33 \mathrm{X}$ complete DMEM-DNase I medium and washed 3 times in complete DMEM-DNase I. The microvessel fraction was resuspended in endothelial cell growth medium [11] (ECGM: DMEM-F12 supplemented with 10\% heat-inac- tivated horse serum, $10 \%$ heat-inactivated fetal calf serum, $100 \mu \mathrm{g} / \mathrm{ml}$ endothelial cell growth supplement (BD Biosciences, Franklin Lakes, NJ), and $100 \mu \mathrm{g} / \mathrm{ml}$ heparin) at a concentration of $\sim 1 \times 10^{4}$ vessel fragments $/ \mathrm{ml}$ and plated on tissue culture dishes coated with murine collagen type IV (BD Biosciences, Franklin Lakes, NJ).

The purity of the resulting EC cultures were assessed based on the capacity of EC to take up acetylated-LDL $[11,12]$. Primary EC cultures grown to $\sim 70 \%$ confluency in ECGM were labeled overnight with $5 \mu \mathrm{g} / \mathrm{ml}$ acetylated low density lipoprotein conjugated with 1,1'-dioctadecyl - 3,3,3',3'-tetramethyl-indocarbocyanine perchlorate (DiIAcLDL, Biomedical Technologies, Stoughton, MA). The culture was then washed extensively with PBS and trypsinized to remove non-internalized DiIAcLDL, and culture purity was assessed by fluorescence-activated cell sorting (FACS). For some experiments the DiIAcLDL labeled EC were collected and expanded in ECGM on glass slides coated with murine collagen type IV and used for direct co-cultures with neural progenitor cells.

\section{Co-cultures of neural progenitor and vascular endothelial} cells.

Long-range interactions were investigated by using transwell co-culture systems similar to those utilized by Shen et al. [10]. In the first experimental system neural progenitor cells from E13.5-14.5 mouse cortex $\left(4 \times 10^{5}\right)$ were seeded into the lower transwell (coated with poly-D-lysine) and simultaneously the upper transwell compartment coated with murine collagen type IV and containing $0.4 \mu \mathrm{m}$-diameter pores was seeded with the endothelial cell preparation $\left(1 \times 10^{4}\right.$ vessel fragments). Both cell types were cultured in endothelial cell growth medium (ECGM) for $72 \mathrm{~h}$ and then switched to Neurobasal medium with B27 supplement (complete Neurobasal) to favor neural differentiation. In the second system, EC alone were established on the upper transwell compartment in ECGM. After $5 \mathrm{~d}$, the medium was switched to complete Neurobasal medium for $24 \mathrm{~h}$. The upper transwell containing the EC, was subsequently placed on top of a poly-D-lysine coated lower chamber freshly seeded with neural progenitor cells and the cultures were maintained in complete Neurobasal medium. To examine direct interactions, neural progenitor and $\mathrm{EC}$ fractions were mixed in ECGM $\left(10^{5}\right.$ neural progenitor cells $/ 10^{3}$ cerebral vessel fragments), and plated on murine collagen type IVcoated dishes in ECGM medium. For some experiments, neural progenitor cells were co-cultured directly with EC cultures that had been purified by FACS after labeling with DiIAcLDL.

\section{Statistical procedures}

Data are presented as mean \pm the standard error of the 
mean (SEM). Statistical comparisons were made using oneway analysis of variance (ANOVA) with Tukey-Kramer post-hoc tests. Statistical tests were performed using the program GraphPad Prism 4.0 (GraphPad Software Inc., San Diego CA).

\section{Results}

Characterization of embryonic vascular endothelial cells (EC).

To study how neural cells and EC interact, we first developed a method for the simultaneous isolation of primary EC and neural progenitor cells from embryonic mouse brain (E13.5-16). This method utilizes a mild trypsinization of embryonic brain that permits the sieve separation of neural cells and the subsequent liberation of EC from cerebral vessels with a gentle collagenase/dispase treatment. The endothelial cell fraction obtained by this method showed positive immunostaining for platelet endothelial cell adhesion molecule c-1 (PECAM, CD31), von Willebrand factor (VWF), fibronectin and laminin (Figure 1). In addition FACS analysis demonstrated that greater than 95\% of the cells internalized acetylated LDL thus confirming their endothelial cell nature (Figure 3 ). On average, about $2 \times 10^{5}$ endothelial cells were obtained from a brain of an E14.5d embryo after 1 week in culture.
Soluble factors from endothelial cells promote maintenance of the neural progenitor state and prevent neural differentiation.

To investigate whether soluble factors from primary EC cultures may affect neural progenitor behavior, we used transwell co-culture systems similar to those utilized by Shen et al. [10]. Neural progenitors were seeded onto poly-D-lysine coated lower chambers and the EC fraction was plated on the upper transwell compartment coated with murine collagen type IV. Since primary EC could not be established on a collagen IV-coated substrate in the absence of serum, both cell types were cultured in endothelial cell growth medium (ECGM) for $72 \mathrm{~h}$ after which they were switched to complete Neurobasal medium for one week to favor neural differentiation. Under these conditions, the neural progenitor cells in the lower chamber grew mainly in suspension and formed neurospheres that exhibited strong nestin expression and contained no MAP2 + cells (Figure $2 \mathrm{~A})$. Only a small fraction $(<4 \%)$ of the initial cell inoculums grew attached to the substratum. These cells, however, retained their neural progenitor status expressing nestin, and none differentiated into MAP2+ neurons (Figure 2B). In contrast, neural progenitor cells cultured in complete Neurobasal medium without EC in the upper transwell

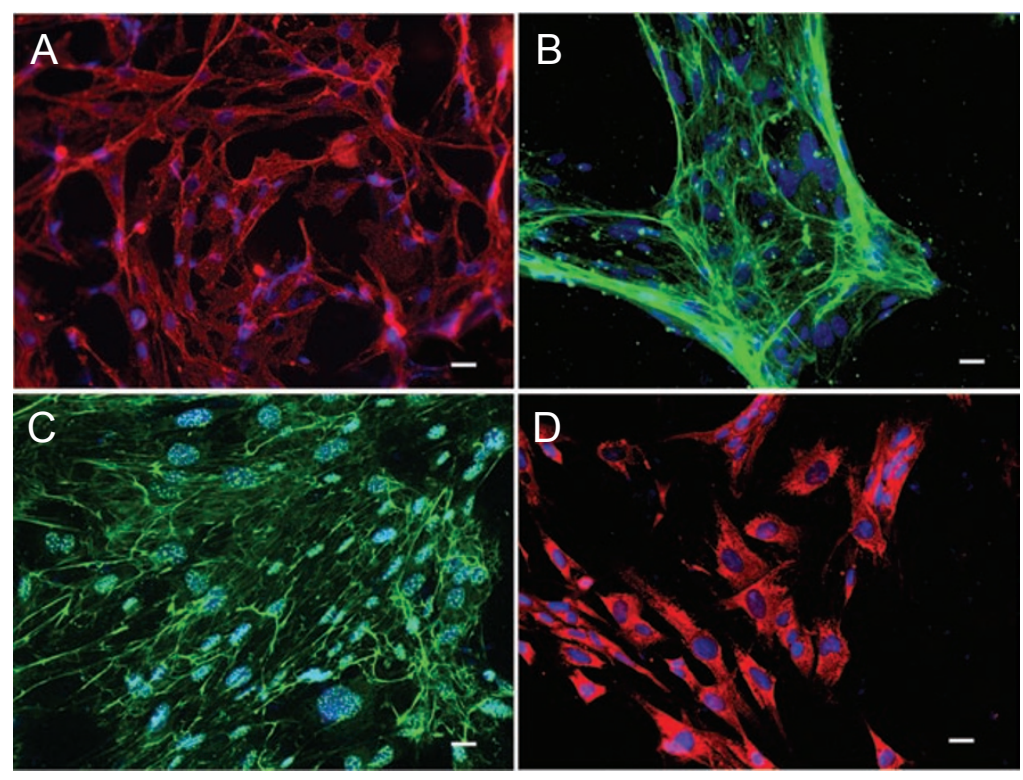

Figure 1 Characterization of embryonic brain primary endothelial cells (EC) with endothelial cell markers. (A) PECAM. (B) von Willebrand Factor (VWF). (C) fibronectin. (D) laminin. Scale: $10 \mu \mathrm{m}$. 

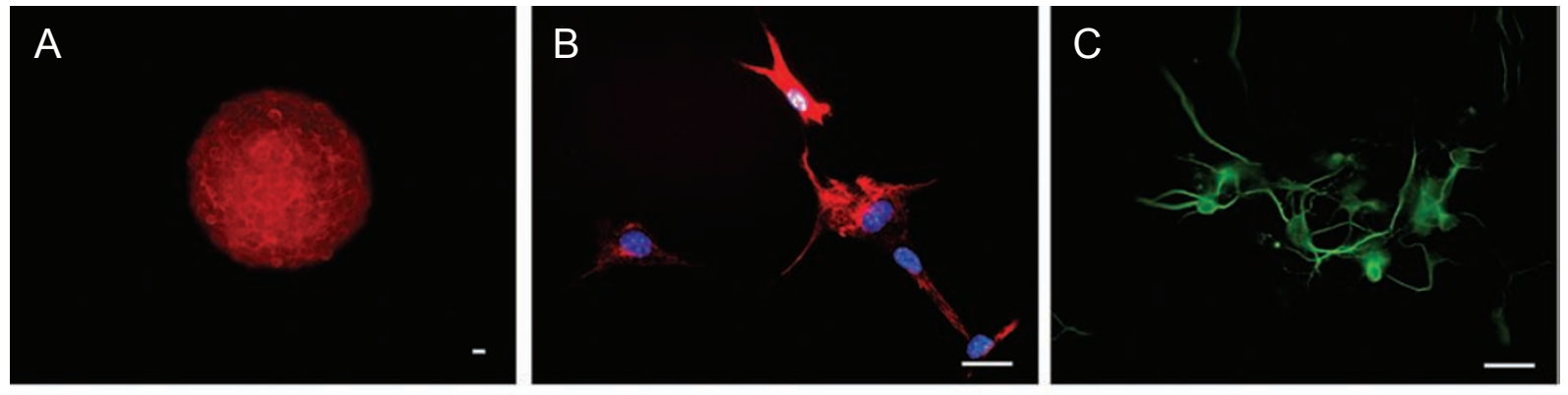

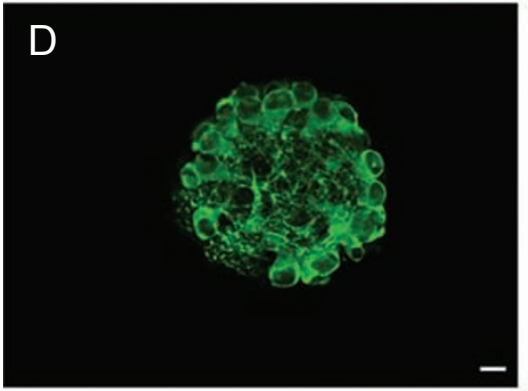

$E$
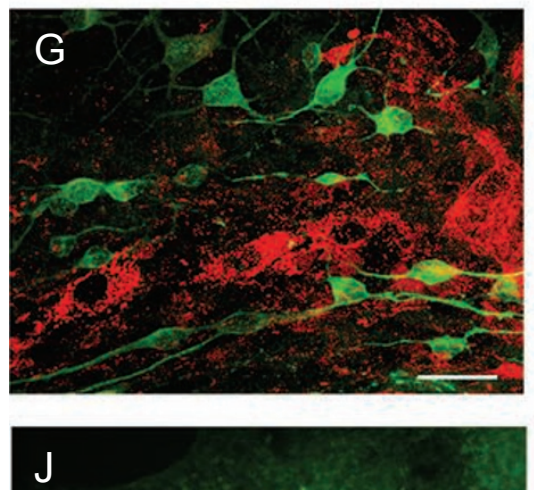

J

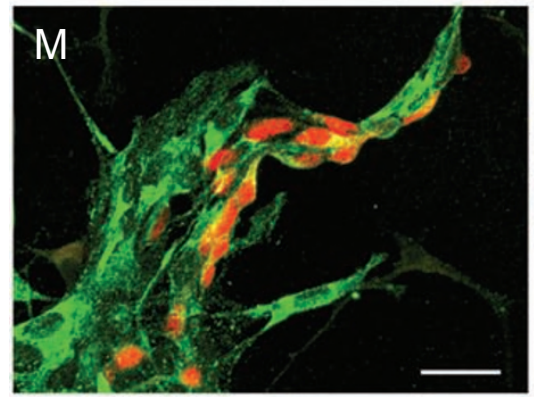

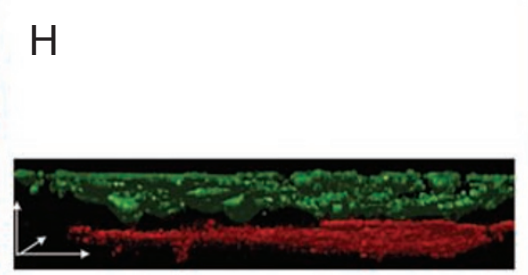
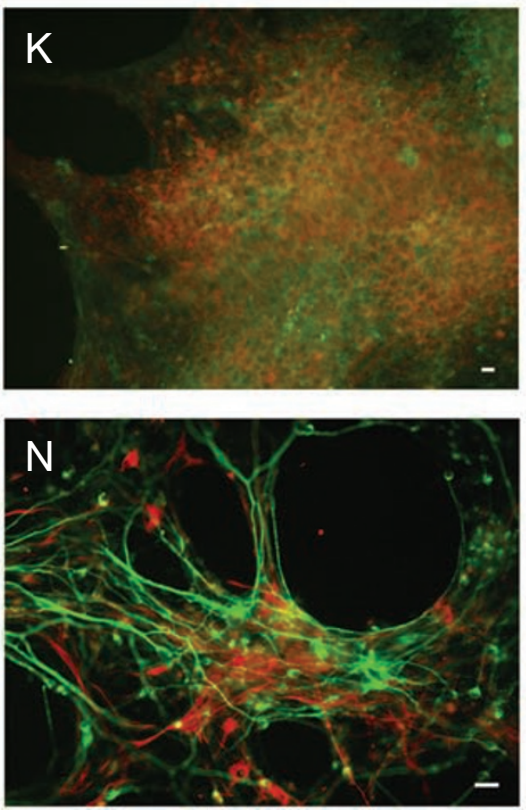
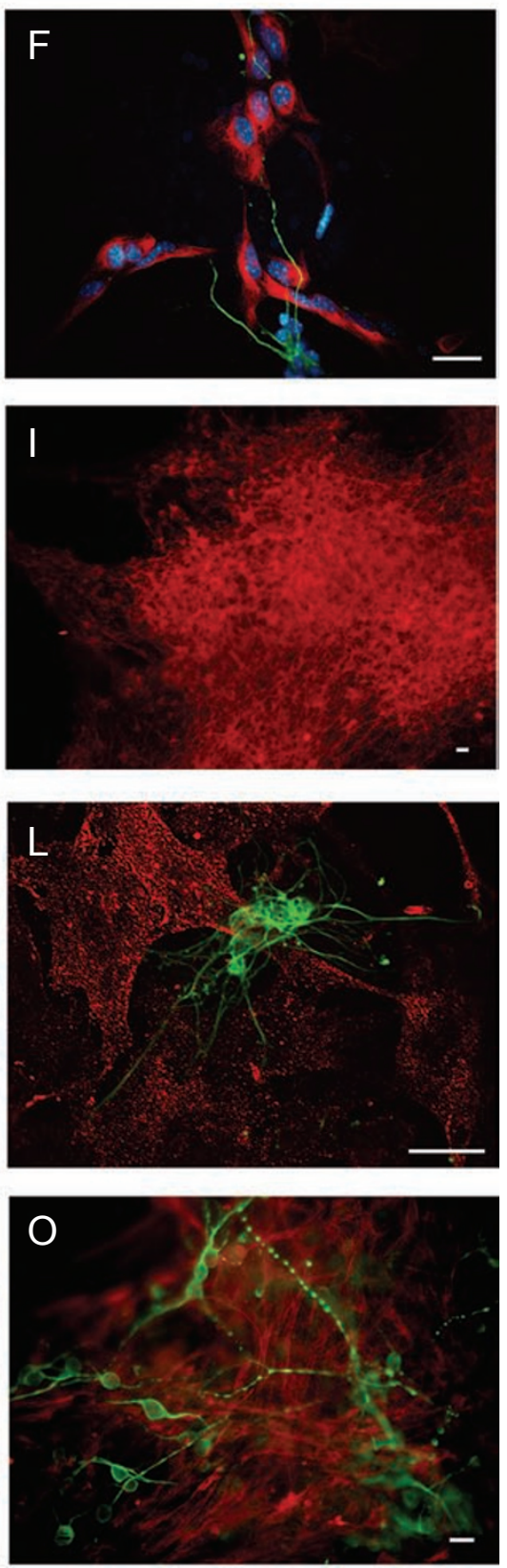

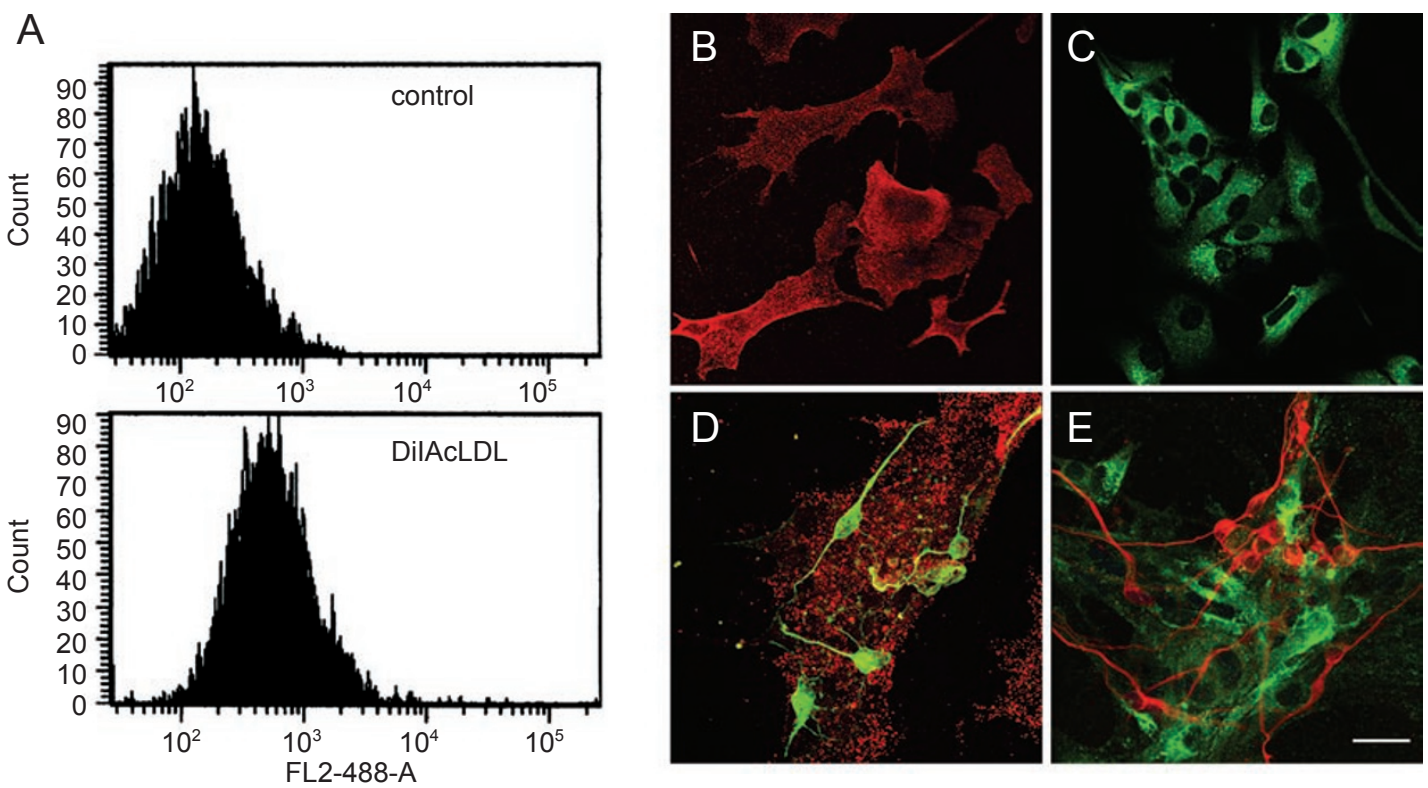

Figure 3 Purity of primary endothelial cell cultures. Panel A: FACS analyses of primary endothelial cells based on their ability to uptake acetylated LDL (DiI-Ac-LDL). Panels B-C: Immunohistochemical characterization of endothelial cells sorted after DiIAc-LDL uptake. (B) PECAM (red). (C) laminin (green). Panels D-E: Contact-induced differentiation of neural progenitor cells by FACS-purified endothelial cells. (D) PECAM (red), MAP2 (green). (E) laminin (green), $\beta$-III tubulin (TuJ, red). Scale: $10 \mu \mathrm{m}$.

readily differentiated into MAP2+ neurons (Figure 2C). Therefore, despite culture conditions that should have promoted neuronal differentiation (poly-D-lysine substrate and complete Neurobasal medium), the neural progenitor cells remained undifferentiated in the presence of EC. To determine whether the neurospheres that developed could be induced to differentiate, neurospheres from the co-cultures were plated on poly-D-lysine-coated coverslips in complete Neurobasal medium. The neurospheres attached to the substrate and differentiated into many MAP2 + neurons after $5 \mathrm{~d}$ in culture (Figure 2D).

Since primary EC could not be established in media

Figure 2 Interactions between neural progenitor and endothelial cells. Panels A-F: Inhibition of neuronal differentiation by soluble EC factors. Transwell co-culture of neural progenitors and EC for $72 \mathrm{~h}$ in ECGM followed by culturing in complete Neurobasal medium. (A) Nestin+ (red) neurosphere (MAP2 green). (B) Nestin+ (red) attached cells (DAPI-stained nuclei in blue, MAP2 green). (C) Neuroprogenitors differentiated into MAP2+ neurons (green) in the absence of EC in the transwell. (D) Neurosphere cultured in the transwell system as in (A) can differentiate into MAP2+ neurons (green) when cultured on poly-D-lysine in complete Neurobasal medium in the absence of EC. (E-F) Neural progenitor and cerebral endothelial cell (EC) fractions were cultured separately in a transwell system in which the neuroepithelial cells were cultured exclusively in complete Neurobasal medium and were not exposed to serum. Examples of nestin+ (red) and MAP2+ (green) attached cells (DAPI-stained nuclei in blue) are shown. Panels G-M: Direct co-culture of neural progenitor cells and brain EC on collagen type IV in ECGM. (G) Confocal microscopy of cells stained with anti-laminin (red) and anti-MAP2 (green) antibodies. (H) Computer-assisted (Neuronstudio software, MBF Biosciences, Williston, VT) 3-dimensional reconstruction of panel (G) showing that MAP2+ neurons (green) differentiated on top of laminin+ EC (red), (see supplementary data for details). Panels I-K: Induced differentiation of neural progenitor cells by direct contact with primary EC. (I) MAP2 (red). (J) laminin (green). (K) merged image. (L) Confocal microscopy of cells stained with antibodies against PECAM (red) and neuronal-specific $\beta$-III tubulin (TuJ, green). (M) NeuN expression in the nuclei of differentiated neurons (red) in direct co-cultures of neural progenitors and laminin+EC (green). (N) Immunostaining with anti-nestin (red) and anti-MAP2 (green) antibodies of neuroprogenitors plated on collagen type IV in the presence of ECGM medium. (O) Nestin (red) and MAP2 (green) immunostaining of neuroprogenitors plated on collagen type IV in ECGM and co-cultured with EC using the transwell system. Scale: $10 \mu \mathrm{m}$. 
lacking serum, such as complete Neurobasal medium, the co-culture experiments described above were initially conducted in the continuous presence of serum. However, the three-day exposure of neural progenitor cells to serum complicates the interpretation of these studies since additional factors found in serum might also be affecting the neural progenitor's state. In order to circumvent this problem, we first established primary EC cell cultures in ECGM on the upper well of a transwell culture plate. After $5 \mathrm{~d}$, the ECGM was replaced with complete Neurobasal medium in order to remove the effects of serum. One day later the upper chamber containing the $\mathrm{EC}$ was transferred onto the top of a new transwell chamber containing freshly isolated neural progenitors plated in the lower chamber, and the cultures were maintained in complete Neurobasal medium. Thus the neural progenitors were exposed to EC factors without being cultured in serum-containing media. Under these conditions the neural progenitor cell fraction proliferated mainly as nestin-positive cells attached to

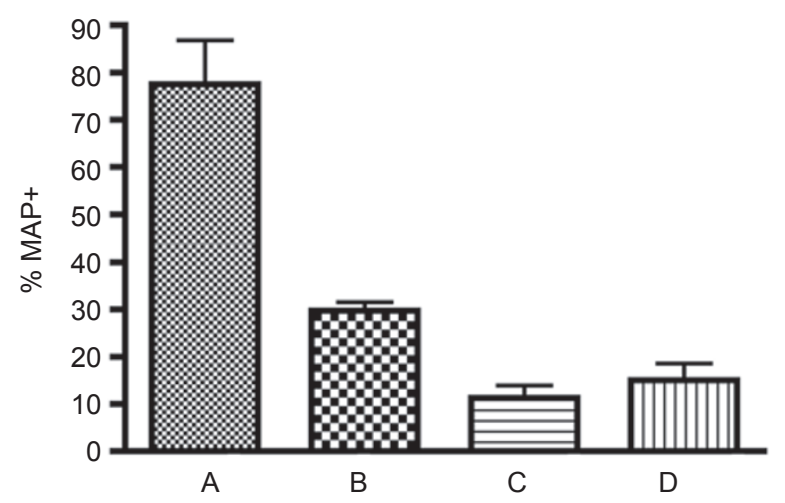

Figure 4 Effects of endothelial cell factors on neural differentiation. (A) Co-culture of endothelial cells and neural progenitors grown in direct contact on collagen IV with ECGM. (B) Neural progenitor cells grown on collagen IV with ECGM. (C) Co-culture of endothelial cells and neural progenitors indirectly in transwell chambers coated with collagen IV and ECGM. (D) Co-culture of endothelial cells grown in the upper transwell chamber coated with collagen IV and neural progenitors in the lower chamber coated with poly-D-lysine in complete Neurobasal medium. Percent (\%) of MAP2+ cells (MAP2+ cells/DAPI+ nuclei) represent the mean of $n=7$ independent experiments for $\mathbf{A}, n=4$ for $\mathbf{B}$ and $\mathbf{C}, n=6$ for $\mathbf{D}$. At least 200 cells were counted per experiment. When MAP2+ cells were determined in direct co-cultures of endothelial cells and neural progenitor cells, the cultures were double labeled with MAP2/laminin and the laminin+ EC were excluded from the analyses. A one-way ANOVA $\left(\mathrm{F}_{3,19}=\right.$ $41.42, p<0.0001)$ with Tukey-Kramer post-hoc tests showed statistically significant differences between $\mathbf{A} v s \mathbf{B}, \mathbf{C}$ and $\mathbf{D}(p<0.001)$ and between $\mathbf{B} v s \mathbf{C}$ and $\mathbf{D}(p<0.05)$. Differences between $\mathbf{C} v s \mathbf{D}$ were not statistically significant. the poly-D-lysine coated-surface (Figure 2E-F, also see supplementary data) and only about $15 \%$ (Figure 4 ) of the cells differentiated into MAP2+ neurons. Therefore, results from both transwell co-culture systems support the notion that soluble factors from primary brain EC promote the maintenance of the neuroprogenitor phenotype.

\section{Direct physical interaction of endothelial cells with neuroprogenitors induces neuronal differentiation and maturation.}

To examine in vitro the effects of direct physical interactions between endothelial cells and neural progenitors we established conditions to co-culture both cell fractions. We found that EC and neuroprogenitors could be mixed and cultured together when plated on collagen type IV and maintained in ECGM. Interestingly we found that the cell attachment to the substrate was not random, as the EC attached to the collagen-coated surface, while the neural progenitor cells preferentially attached to the EC and formed a top cellular layer that expressed the neuronal markers MAP2, $\beta$ III-tubulin (TuJ) and NeuN (Figure 2G-M, also see video in supplementary data). Within one week, about $78 \%$ of the neuroprogenitor cells (distinguished from EC by their lack of laminin staining) had differentiated into MAP2 immunoreactive neurons (Figure 4).

To minimize the chance that a non-endothelial cell type was responsible for inducing neuronal differentiation, DiIAcLDL-labelled endothelial cells were purified by FACS and co-cultured in contact with neuroprogenitor cells in ECGM (Figure 3A). The purified endothelial cell fraction, which was $>99 \%$ PECAM and laminin positive (Figure 3B-C), induced a comparable degree of neuronal differentiation when co-cultured with neural progenitors (Figure 3D-E). Thus when cultured in direct contact with one another, EC form a scaffold onto which neural progenitors attach and this direct physical contact promotes neuronal differentiation.

Among the molecules that could mediate such interactions, collagen type IV, a component of the vascular basal lamina produced by EC, is an interesting candidate since it has been reported to inhibit the proliferation of rat neural progenitors and promote their differentiation into neurons [13]. We determined whether neural progenitor cells could be induced to differentiate on a collagen type IV substrate in the absence of EC. We initially attempted to culture neural progenitors on collagen type IV-coated dishes in complete Neurobasal medium. However, these culture conditions did not support long-term cellular survival. Yet, when plated and maintained in ECGM, the neural progenitor cells not only attached to the collagen type IV surface, but after $7 \mathrm{~d}$ in culture about $30 \%$ of the cells had differentiated into MAP2 immunoreactive neurons (Figure 2N, Figure 
4). While these results suggest that type IV collagen may provide some stimulus for neural progenitor differentiation, the number of MAP2 positive cells is significantly less than that seen in direct co-cultures of neural progenitors and endothelial cells. This suggests that while collagen type IV (expressed in the proper context) may be involved in EC-induced neuronal differentiation, additional factors are needed to see the full effects of EC on neural progenitor differentiation.

We also determined whether a collagen type IV substratum could override the effect of the EC-derived soluble factors that promote maintenance of the undifferentiated state. For these studies we repeated the transwell experiments as described above, in which EC and neural progenitor cells were simultaneously plated in transwell chambers except that neural progenitors were plated on collagen type IVcoated lower chambers and the cultures were maintained in ECGM throughout the experiment. Interestingly under these conditions the neural progenitors attached to the collagen substrate and established monolayer cultures. After one week in culture $80-90 \%$ of the attached cells retained expression of the neural progenitor marker nestin and only about $11 \%$ differentiated into MAP 2 immunopositive neurons (Figure 2O, Figure 4). These results suggest that a collagen type IV substrate alone is unable to completely override the effects of EC-derived soluble factors that promote maintenance of the neural progenitor state and prevent neural differentiation.

\section{Discussion}

Disruption of neurovascular networks is known or thought to play a pathogenic role in diseases as diverse as acute stroke, vascular dementia, multiple sclerosis, cerebral amyloid angiopathy and peripheral neuropathy, as well as in changes associated with normal aging [5, 14]. Given the importance of vascular and neural interactions in development and also in pathophysiology, it is essential to develop primary cell culture systems to better understand such interactions. These systems must include both neural and endothelial cell populations, which should maintain as much as possible their in vivo properties. Although culturing primary neurons as adherent cells or neural progenitor cells as neurospheres is now common, the culture of brain EC has been more challenging. Several protocols have been described for the isolation and propagation of primary brain EC from mouse $[11,15]$. These methods have in general utilized postnatal (P10) brain tissue slices or homogenates that are treated with collagenase/dispase to liberate the vascular EC, which are subsequently grown in media supplemented with growth factors [11, 15]. Although useful these methods have not been widely adopted, likely due to their low yields. As such, most studies characterizing neural/endothelial cell interactions continue to rely on immortalized cell lines that may exhibit altered properties due to their in vitro derivation and passage $[10,16]$.

To study neurovascular interactions in vitro, we developed a method for the simultaneous isolation of mouse neural progenitor and vascular endothelial cells from embryonic brain. The cultured EC cells were $>95 \%$ pure and expressed typical endothelial markers such as PECAM, laminin, fibronectin and VWF and were able to uptake acetylated-LDL. Although used as primary cultures in the experiments presented here, we have passed these cells at high densities ( $>15$ passages, 1:2 dilution) without losing the expression of the EC markers tested.

Using primary cultures isolated with this method we found that $\mathrm{EC}$ affect neural progenitors differently depending on whether they are in direct or indirect contact with neural progenitors. When contact is indirect, EC produce soluble factors that promote neural progenitor expansion and prevent neural differentiation as originally described by Shen et al. [10]. Our findings with primary EC provide additional evidence that endothelial cells play a role in affecting neural progenitor behavior through secreted soluble factors.

We also addressed how EC affect neural progenitors when grown in direct contact. To do so we had to first establish culture conditions that allowed their co-culture and found that co-cultures were possible if both cell types were plated on a collagen type IV-coated substrate and maintained in ECMG. Under these conditions the cells formed two distinct layers with the EC attaching preferentially to the substrate and the neural progenitor cells attaching preferentially on top of the EC (Figure 2H). Direct contact provided in this manner resulted in neural progenitor cell differentiation into neurons.

Vascular development is generally divided into two distinct processes, termed vasculogenesis and angiogenesis $[17,18]$. Vasculogenesis refers to the coalescence of separated angioblasts into endothelial tubes while angiogenesis refers to the de novo formation of blood vessels by the sprouting and splitting of vessels already established by vasculogenesis. Blood vessel formation in the early embryo initially occurs through vasculogenesis including the formation of a capillary network in the head mesenchyme. The brain vascular system develops from the cephalic mesenchyme by the sprouting of capillaries into the brain parenchyma, a process that is regarded as primarily angiogenic [1]. The results of the in vitro experiments reported here suggest that as nascent blood vessels grow into the brain, soluble factors secreted by EC may function as a long distance signal to favor neural progenitor selfrenewal and inhibit their differentiation. However, as EC 
and neural progenitors come into contact, differentiation may be promoted through direct cell-cell interactions. It will be interesting to further characterize the role of specific signaling molecules (on the neuronal and EC sides) that are involved in such interactions. The isolation method and co-culture systems described here should represent versatile in vitro tools for pursuing such questions.

\section{Acknowledgements}

This work was supported by a Merit Award from the Department of Veterans Affairs and grants AG02219 and AG05138 from the National Institutes of Health (NIH, USA). Rita De Gasperi is a recipient of a Young Investigator Award from the National Alliance for Research in Schizophrenia and Affective Disorders (NARSAD). We would like to thank our collegues from the Mount Sinai School of Medicine Drs. Susan Wearne, Alfredo Rodriguez and Douglas Elhenberger for the Neurostudio software for 3D reconstruction; and Dr. Robert Hennigan and Ms. Rumana Huq for their help in the microscopic analyses. Confocal laser scanning microscopy was performed at the MSSMMicroscopy Shared Resource Facility, supported with funding from the National Institutes of Health-National Cancer Institute (NIH-NCI, USA) shared resources grant 5R24 CA095823-04, National Science Fundation (NSF, USA) Major Research Instrumentation grant DBI-9724504 and NIH shared instrumentation grant 1 S10 RR0 9145-01.

\section{References}

1 Harrigan MR. Angiogenic factors in the central nervous system. Neurosurgery 2003; 53:639-660.

2 Autiero M, De Smet F, Claes F, Carmeliet P. Role of neural guidance signals in blood vessel navigation. Cardiovasc Res 2005; 65:629-638.

3 Eichmann A, Le Noble F, Autiero M, Carmeliet P. Guidance of vascular and neural network formation. Curr Opin Neurobiol 2005; 15:108-115.

4 Eichmann A, Makinen T, Alitalo K. Neural guidance molecules regulate vascular remodeling and vessel navigation. Genes Dev
2005; 19:1013-1021.

5 Park JA, Choi KS, Kim SY, Kim KW. Coordinated interaction of the vascular and nervous systems: from molecule-to cell-based approaches. Biochem Biophys Res Commun 2003; 311:247253.

6 Storkebaum E, Lambrechts D, Carmeliet P. VEGF: once regarded as a specific angiogenic factor, now implicated in neuroprotection. Bioessays 2004; 26:943-954.

7 Ward NL, LaManna JC. The neurovascular unit and its growth factors: coordinated response in the vascular and nervous systems. Neurol Res 2004; 26:870-883.

8 Raab S, Beck H, Gaumann A, et al. Impaired brain angiogenesis and neuronal apoptosis induced by conditional homozygous inactivation of vascular endothelial growth factor. Thromb Haemost 2004; 91:595-605.

9 Alvarez-Buylla A, Lim DA. For the long run: maintaining germinal niches in the adult brain. Neuron 2004; 41:683-686.

10 Shen Q, Goderie SK, Jin L, et al. Endothelial cells stimulate selfrenewal and expand neurogenesis of neural stem cells. Science 2004; 304:1338-1340.

11 Song L, Pachter JS. Culture of murine brain microvascular endothelial cells that maintain expression and cytoskeletal association of tight junction-associated proteins. In Vitro Cell Dev Biol Anim 2003; 39:313-320.

12 Voyta JC, Via DP, Butterfield CE, Zetter BR. Identification and isolation of endothelial cells based on their increased uptake of acetylated-low density lipoprotein. J Cell Biol 1984; 99:20342040.

13 Ali SA, Pappas IS, Parnavelas JG. Collagen type IV promotes the differentiation of neuronal progenitors and inhibits astroglial differentiation in cortical cell cultures. Dev Brain Res 1998; 110:31-38.

14 Lo EH, Dalkara T, Moskowitz MA. Mechanisms, challenges and opportunities in stroke. Nat Rev Neurosci 2003; 4:399-415.

15 Jung SS, Levy E. Murine cerebrovascular cells as a cell culture model for cerebral amyloid angiopathy: isolation of smooth muscle and endothelial cells from mouse brain. Methods Mol Biol 2005; 299:211-219.

16 Schiera G, Sala S, Gallo A, et al. Permeability properties of a three-cell type in vitro model of blood-brain barrier. J Cell Mol Med 2005; 9:373-379.

17 Carmeliet P. Mechanisms of angiogenesis and arteriogenesis. Nat Med 2000; 6:389-395.

18 Risau W. Mechanisms of angiogenesis. Nature 1997; 386:671674.

(Supplementary Information is linked to the online version of the paper on the Cell Research website.) 